\title{
Seleção de Carteiras Utilizando o Modelo Fama-French-Carhart
}

\author{
João F. Caldeira*, Guilherme V. Moura ${ }^{\dagger}$, André A. P. Santos ${ }^{\ddagger}$
}

\author{
Conteúdo: 1. Introdução; 2. 0 Modelo; 3. Aplicação em Otimização de Carteiras; \\ 4. Considerações Finais; 5. Apêndice 1: Construção dos Fatores de \\ Fama-French-Carhart; 6. Apêndice 2: Modelos de Volatilidade Univariados. \\ Palavras-chave: Correlação Condicional Dinâmica (DCC); Previsão; Filtro de Kalman; CAPM com \\ Aprendizagem; Otimização de Carteiras; Avaliação do Desempenho. \\ Códigos JEL: $\quad$ C53; E43; G17.
}

Neste artigo utiliza-se o modelo fatorial Fama-French-Carhart para obter portfólios ótimos de mínima variância irrestritos e restritos para vendas a descoberto. Para esse propósito, matrizes de covariâncias condicionais são obtidas com base em uma recente especificação GARCH fatorial multivariada proposta por Santos e Moura (2012) a qual adota uma modelagem flexível para os fatores comuns, para os ativos individuais, e para os pesos dos fatores. Uma aplicação envolvendo 61 ações negociadas na Bolsa de Valores de São Paulo (BM\&FBovespa) mostrou que a especificação proposta gera portfólios menos arriscados fora da amostra em comparação com várias especificações benchmark, incluindo modelos fatoriais existentes.

In this article the Fama-French-Carhart factor model is used to obtain short selling-constrained and unconstrained minimum variance portfolios. For that purpose, conditional covariance matrices are obtained based on a recent multivariate factor GARCH specification with a flexible modeling strategy for the common factors, for the individual assets, and for the factor loads proposed by Santos e Moura (2012). An application involving 61 stocks traded on the São Paulo stock exchange (BMEFBovespa) shows that the proposed specification delivers less risky portfolios on an out-of-sample basis in comparison to several benchmark models, including existing factor approaches.

\footnotetext{
*Departmento de Economia - Universidade Federal do Rio Grande do Sul. E-mail: joao.caldeira@ufrgs .br ${ }^{\dagger}$ Departmento de Economia - Universidade Federal de Santa Catarina. E-mail: guilherme .moura@uf sc .br

${ }^{\ddagger}$ Departmento de Economia - Universidade Federal de Santa Catarina. E-mail: andre .portela@ufsc .br.
} 


\section{INTRODUÇÃO}

O processo de seleção de carteiras de investimento em ativos de risco permanece como um dos problemas centrais em economia financeira, tanto do ponto de vista acadêmico como também para os praticantes de mercado. Neste contexto, a utilização de modelos quantitativos tem ganhado notoriedade em virtude de sua aplicabilidade prática nos processos de alocação e gestão de carteiras de investimento. Boa parte desses modelos estão construídos sob o paradigma da análise média-variância introduzida por Markowitz (1952), a qual transformou o processo de alocação de ativos em um problema de otimização com base no trade-off fundamental entre retorno esperado e risco. Dessa forma, o investidor deve escolher a carteira com a menor variância entre um infinito número de carteiras que proporcionassem um determinado retorno ou, de forma equivalente, para um determinado nível de aversão ao risco, escolher a carteira que maximize o retorno esperado.

Na prática, a implementação da otimização de carteiras com base no critério média-variância esbarra na dificuldade de se obter estimações acuradas dos retornos esperados dos ativos e da matriz de covariâncias desses retornos. Via de regra, estas estimativas estão sujeitas a erros de estimação, o que prejudica o desempenho da carteira otimizada com base nestes estimadores. Além disso, a estimação de retornos esperados está sujeita a um maior erro de estimação em comparação à estimação de covariâncias (Merton, 1980, Jagannathan e Ma, 2003), e o erro de estimação nos retornos esperados tem um impacto negativo maior no desempenho da carteira quando comparado ao impacto do erro de estimação nas covariâncias (Michaud, 1989, Best e Grauer, 1991, Mendes e Leal, 2005, Ceria e Stubbs, 2006). Em vista disso, a pesquisa acadêmica recente tem focado em carteiras de mínima variância, a qual depende somente da estimação de covariâncias e estão sujeitas a uma quantidade menor de erro de estimação em comparação às carteiras de média-variância (DeMiguel et alii, 2009a). Neste caso, o investidor importa-se apenas com a minimização do risco da carteira, sem levar em consideração seu retorno esperado. De fato, uma extensa lista de referências tem apontado que carteiras de mínima variância possuem um desempenho fora da amostra melhor que quaisquer carteiras de média-variância, mesmo quando o critério de desempenho considera tanto o retorno da carteira como também seu risco; a esse respeito ver, por exemplo, Ledoit e Wolf (2004), Engle e Sheppard (2008), DeMiguel et alii (2009a), Santos e Moura (2012) e Santos e Tessari (2012), dentre outros.

Outra dificuldade diz respeito ao problema da dimensionalidade, uma vez que é frequente a necessidade de se obter carteiras otimizadas contendo um número muito grande de ativos. A maioria das tentativas iniciais de se construir modelos para as covariâncias condicionais como, por exemplo, o modelo VEC de Bollerslev et alii (1988) e o modelo BEKK de Engle e Kroner (1995), entre outros, sofrem da chamada maldição da dimensionalidade. Nestas especificações, o número de parâmetros cresce rapidamente a medida que a dimensão do problema aumenta, criando dificuldades no processo de estimação e introduzindo erro de estimação nas matrizes de covariâncias estimadas. Neste contexto, modelos de fatores surgem como uma alternativa promissora para contornar o problema da dimensionalidade e aliviar a carga do processo de estimação econométrica. A ideia dos modelos de fatores é assumir que os movimentos dos retornos financeiros dependem de um pequeno número de variáveis subjacentes chamadas fatores. Esta redução de dimensionalidade permite uma grande flexibilidade na especificação econométrica e na estratégia de estimação. De fato, diferentes abordagens para as matrizes de covariâncias condicionais baseadas em modelos de fatores tem sido propostas na literatura. Geralmente, estes modelos diferem em suas suposições a respeito das características dos fatores. Por exemplo, Alexander e Chibumba (1996) e Alexander (2001) obtém fatores comuns a partir de técnicas estatísticas como análise de componentes principais (PCA), enquanto Chan et alii (1999) utilizam fatores comuns extraídos a partir dos retornos dos ativos. Engle et alii (1990), Alexander e Chibumba (1996), Alexander (2001) e Vrontos et alii (2003) assumem que os fatores seguem um processo GARCH, enquanto Aguilar e West (2000) e Han (2006) consideram uma dinâmica com base num modelo de volatilidade estocástica.

Neste artigo utiliza-se a extensão proposta por Carhart (1997) do modelo fatorial de apreçamento de ativos de Fama e French (1996) (aqui chamado modelo Fama-French-Carhart) para a obtenção de 
carteiras ótimas de mínima variância (CMV) utilizando uma amostra de ações negociadas no mercado brasileiro. Esta especificação encontra amplo respaldo na literatura especializada, uma vez que logra capturar a maior parte das anomalias não assimiladas pelo modelo de apreçamento de ativos de capital CAPM (Brav et alii, 2000, Ekbo et alii, 2000, Jegadeesh, 2000, Liew e Vassalou, 2000, Costa Jr e Neves, 2000, Chung e Schill, 2006, Malaga e Securato, 2004, Mussa et alii, 2012). Além de levar em conta a exposição do ativo em relação a carteira de mercado, o modelo Fama-French-Carhart considera como fatores de risco o tamanho da empresa, definido pelo valor de mercado do patrimônio líquido, o índice book-to-market ou BE/ME, definido pela relação entre o valor contábil e de mercado do patrimônio líquido e o fator momento, dado pela persistência temporal do desempenho dos retornos das ações.

Como forma de obter matrizes de variâncias condicionais para problemas envolvendo um grande número de ativos, emprega-se neste artigo uma especificação econométrica proposta recentemente por Santos e Moura (2012), a qual baseia-se em um modelo de fatores dinâmicos heterocedástico e flexível (este modelo será denotado pela abreviação DFGARCH). A metodologia proposta por Santos \& Moura estende as especificações econométricas prévias em ao menos dois aspectos. Primeiro, a abordagem proposta é bastante flexível permitindo o uso de diferentes especificações econométricas para os fatores comuns e para os ativos individuais em um portfólio. Em particular, o modelo permite uma especificação multivariada parcimoniosa para as covariâncias entre os fatores com base em um modelo de correlação condicional dinâmica e considera diferentes especificações GARCH univariadas para modelar a volatilidade dos ativos individuais. Além disso, os pesos dos fatores são tratados como variáveis latentes com uma dinâmica baseada em recentes desenvolvimentos na teoria de apreçamento de ativos (Adrian e Franzoni, 2009).

O modelo DFGARCH de Santos e Moura (2012) é aplicado para obter previsões um passo à frente dentro e fora da amostra para as matrizes de covariância de 61 ações negociadas no mercado brasileiro durante o período amostral. Essas matrizes são usadas para estimar CMV irrestritas e com restrição de venda a descoberto. $\mathrm{O}$ desempenho do modelo proposto é comparado àquela obtida por diversos modelos benchmark alternativos, incluindo modelos fatoriais existentes. Os resultados indicam que o modelo proposto resulta em carteiras com menor nível de risco em comparação aos modelos benchmark, além de apresentar melhor desempenho em termos de retornos ajustados pelo risco na maioria dos experimentos fora da amostra. Vale pontuar também que a aplicação empírica realizada neste artigo envolve a estimação de matrizes de covariâncias condicionais para 61 ativos, o que está muito acima do número de ativos utilizado na maioria das aplicações empíricas existentes que utilizam dados brasileiros. Desta forma, esta aplicação realizada com dados brasileiros é pioneira no sentido de considerar a estimação de matrizes de covariâncias condicionais com esta dimensão. Estudos empíricos anteriores para o mercado brasileiro como, por exemplo, Thomé Neto et alii (2011) e Santos e Tessari (2012), costumam adotar uma abordagem incondicional para o cálculo das matrizes de covariâncias e um número menor de ativos nas suas aplicações empíricas.

$O$ artigo é organizado como segue. Na Seção 2 descrevemos a especificação do modelo e fornecemos detalhes da estimação e dos modelos relacionados. Na Seção 3 discutimos uma aplicação no contexto de otimização de carteiras e propomos uma metodologia para avaliação do desempenho para fora da amostra. Finalmente, a Seção 4 apresenta uma síntese conclusiva do artigo.

\section{O MODELO}

A extensão do modelo de três fatores de Fama e French (1993) proposta por Carhart (1997), em sua representação de séries temporais, pode ser expresso da seguinte forma:

$$
y_{i t}=\alpha_{i t}+\beta_{1 i t}\left(R_{m}-R_{f}\right)+\beta_{2 i t} S M B_{t}+\beta_{3 i t} H M L_{t}+\beta_{4 i t} P R 1 Y R_{t}+\varepsilon_{i t}
$$

onde $R_{m}-R_{f}$ representa o excesso de retorno de um carteira composta por todas as ações que fizeram parte do índice Ibovespa durante o período amostral, ponderada pelo valor de mercado (capitalização a 
mercado), sobre a taxa de juros do CDI diário. Em função da limitada capacidade do modelo CAPM em explicar as variações nos retornos das ações, foram adicionados ao modelo fatores que imitam portfólios com o objetivo de resolver os erros de precificação do CAPM. Assim, o primeiro portfólio é construído através da compra de empresas pequenas (small caps) e da venda de empresas grandes (large caps). Este portfólio é conhecido como small-minus-big (SMB). O segundo portfolio envolve a compra de firmas com elevada razão book-to-market e na venda de firmas como baixa razão book-to-market, este portfólio é chamado de high-minus-low (HML). Finalmente, o terceiro portfólio compra ações que performaram bem recentemente e vende ações com fraco desempenho. Portanto, este portfólio aborda o problema de que ações com bom desempenho recente e ações com baixa performance vão persistir no curto prazo. Tal portfólio é chamado de prior 1-year momentum $(P R 1 Y R)$. Note-se que os três portfólios não envolvem investimentos líquidos. Para construir as carteiras, foi utilizada uma metodologia semelhante a de Fama e French (1993) e Carhart (1997). ${ }^{1}$ Além disso, o peso de cada ativo na carteira é determinado pela capitalização a mercado da empresa. $\varepsilon_{i t} \sim N\left(0, h_{i t}\right)$ é o $i$-ésimo erro de medida, e $\beta_{i t}$ é a $i$-ésima linha da matriz contendo os pesos dos fatores $\beta_{t}$ com dimensão $N \times K$, onde $N$ é o número de ativos e $K$ é o número de fatores. Por suposição, os fatores $f_{t}$ são:

i) condicionalmente ortogonais aos erros de medida, $E\left[f_{i t} \varepsilon_{j t} \mid \Im_{t-1}\right]=0 \forall i \in\{1, \ldots, K\} \forall j \in$ $\{1, \ldots, N\}$ e

ii) não são condicionalmente mutuamente ortogonais, i.e, $E\left[f_{i t} f_{j t} \mid \Im_{t-1}\right] \neq 0 \forall i \neq j$, onde $f_{i}$ é o $i$-ésimo fator e $\Im_{t-1}$ denota o conjunto de informações disponíveis até $t-1$.

Além disso, assume-se que os erros de medida são condicionalmente ortogonais à variância condicional variante no tempo, i.e $E\left[\varepsilon_{i t} \varepsilon_{j t} \mid \Im_{t-1}\right]=0 \forall i \neq j$ e $E\left[\varepsilon_{i t} \varepsilon_{j t} \mid \Im_{t-1}\right]=h_{i t} \forall i=j$.

Uma importante característica da especificação em (1) é que os pesos dos fatores são variantes no tempo. As evidências existentes sugerem que permitir que os pesos dos fatores variem ao longo do tempo leva a ganhos em termos de erros de apreçamento e acurácia de previsão; a esse respeito ver, por exemplo, Jostova e Philipov (2005), Ang e Chen (2007), e Adrian e Franzoni (2009). Neste artigo, segue-se a metodologia proposta por Santos e Moura (2012) e assume-se que cada $\beta_{t}$ em (1) evolui de acordo com duas leis de movimento alternativas. A primeira lei de movimento considera que os pesos dos fatores são não-observáveis e seguem um random walk (RW),

$$
\beta_{i t}=\beta_{i t-1}+u_{i t}
$$

onde $u_{i t} \sim N\left(0, \Sigma_{u_{i}}\right)$ é o vetor de erros $1 \times K$ na lei de movimento dos pesos dos fatores, $\varepsilon_{i t}$ e $u_{i t}$ são independentes. A segunda lei de movimento considerada é baseada no modelo de aprendizagem dos fatores proposto por Adrian e Franzoni (2009). No modelo de aprendizagem, o peso dos fatores segue um processo de reversão à média no qual investidores desconhecem seu nível de longo prazo. Consequentemente, no modelo de aprendizagem é preciso inferir o nível corrente do peso do fator e sua média de longo prazo a partir do histórico dos retornos observados. Desta forma, cada $\beta_{i t}$ evolui de acordo com

$$
\begin{aligned}
\beta_{i t} & =(1-\phi) B_{i t}+\phi \beta_{i t-1}+u_{i t} \\
B_{i t} & =B_{i t-1}
\end{aligned}
$$

onde $B_{i t}$ é o valor esperado da média de longo prazo de $\beta_{t}$ no tempo $t, u_{i t} \sim N\left(0, \Sigma_{u_{i}}\right)$ é um vetor de erros $1 \times K$ na lei de movimento dos pesos dos fatores no modelo de aprendizagem. Finalmente, assume-se que $\varepsilon_{i t}$ e $u_{i t}$ são independentes.

A matriz de covariância condicional, $H_{t}$, do vetor de retornos em (1) é dada por

$$
H_{t}=\beta_{t} \Omega_{t} \beta_{t}^{\prime}+\Xi_{t}
$$

\footnotetext{
${ }^{1}$ Para detalhes sobre a construção dos fatores ver Apêndice 1.
} 
onde $\Omega_{t}$ é uma matriz simétrica e positiva definida com as covariâncias condicionais dos fatores e $\Xi_{t}$ é uma matriz diagonal de covariância dos resíduos do modelo fatorial em (1), ou seja, $\Xi_{t}=$ diag $\left(h_{1 t}, \ldots, h_{N t}\right)$, onde diag é um operador que transforma o vetor $N \times 1$ em uma matriz diagonal $N \times N$ e $h_{i t}$ é a variância condicional do resíduo do $i$-ésimo ativo. Note que, por definição, as duas partes do lado direito em (5) são positivas definidas de modo a assegurar que a matriz de covariância, $H_{t}$, seja positiva definida para todo $t$.

Para obter a matriz de covariância condicional dos fatores, $\Omega_{t}$, em (5) podem ser consideradas especificações alternativas, incluindo modelos GARCH multivariados (ver Bauwens et alii (2006) e Silvennoinen e Teräsvirta (2009) para uma revisão mais detalhada) e modelos de volatilidade estocástica (Harvey et alii, 1994, Aguilar e West, 2000, Chib et alii, 2009). Neste artigo é empregado o modelo de correlações condicionais dinâmicas (dynamic conditional correlation - DCC) proposto por Engle (2002), o qual é dado por

$$
\Omega_{t}=D_{t} R_{t} D_{t}
$$

onde $D_{t}=\operatorname{diag}\left(\sqrt{h_{f_{1 t}}}, \ldots, \sqrt{h_{f_{k t}}}\right), h_{f_{k t}}$ é a variância condicional do $k$-th fator e $R_{t}$ é uma matriz simétrica postiva definida das correlações condicionais com elementos $\rho_{i j, t}$, onde $\rho_{i i, t}=1, i, j=$ $1, \ldots, K$. No modelo DCC a correlação condicional $\rho_{i j, t}$ é dada por

$$
\rho_{i j, t}=\frac{q_{i j, t}}{\sqrt{q_{i i, t} q_{j j, t}}}
$$

onde $q_{i j, t}, i, j=1, \ldots, K$, são colocados na matriz $Q_{t}$ de dimensnão $(K \times K)$ à qual assume-se que segue um processo GARCH,

$$
Q_{t}=\left(1-\alpha_{1}-\alpha_{2}\right) \bar{Q}+\alpha_{1} z_{t-1} z_{t-1}^{\prime}+\alpha_{2} Q_{t-1}
$$

onde $z_{f_{t}}=\left(z_{f_{1 t}}, \ldots, z_{f_{k t}}\right)$ com elementos $z_{f_{i t}}=f_{i t} / \sqrt{h_{f_{i t}}}$ sendo o retorno padronizado do fator, $\bar{Q}$ é a matriz de covariância incondicional de $z_{t}$, também $(K \times K), \alpha_{1}$ e $\alpha_{2}$ são parâmetros escalares não negativos satisfazendo $\alpha_{1}+\alpha_{2}<1$.

Assim como em Santos e Moura (2012), neste trabalho adota-se uma estratégia similar à de Cappiello et alii (2006) e considera-se diferentes especificações do tipo GARCH para modelar a variância condicional dos fatores $h_{f_{k t}}$, e a variância condicional dos resíduos, $h_{i t}$. Em particular, utiliza-se o modelo GARCH de Bollerslev (1986), o modelo GJR-GARCH assimétrico de Glosten et alii (1993), o modelo exponencial GARCH (EGARCH) de Nelson (1991), o modelo treshold GARCH (TGARCH) de Zakoian (1994), o modelo exponencial GARCH assimétrico (APARCH) de Ding et alii (1993), o modelo GARCH assimétrico de Engle (1990), e o modelo GARCH não linear assimétrico (NAGARCH) de Engle e Ng (1993). Em todos os modelos é empregada a versão mais simples, em que a variância condicional depende apenas em uma defasagem dos retornos e da variância condicional. No Apêndice 2 são descritas as especificações econométricas de cada um desses modelos.

\subsection{Estimação}

O modelo DFGARCH é estimado através de um procedimento em multi-etapas. Primeiramente, são estimados os pesos dos fatores variantes no tempo em (1) via máxima verossimilhança (ML). Na segunda etapa, são obtidas as matrizes de covariâncias condicionais dos fatores em (6) através um modelo DCC para as séries temporais dos retornos dos fatores, assumindo que as inovações são Gaussianas. Os parâmetros do modelo DCC são estimados usando o método de máxima verossimilhança composta (CL), proposto por Engle et alii (2008). Finalmente, são consideradas especificações GARCH univariadas alternativas para obter as variâncias condicionais dos resíduos do modelo de fatores. Para cada série dos resíduos, escolhe-se a especificação que maximiza o Critério de Informação de Akaike (AIC). Na próxima seção são apresentados mais detalhes dos procedimentos de estimação. 


\section{Estimação dos Pesos dos Fatores Variando no Tempo}

Dados os fatores $f_{t}$, os sistemas de equações (1)-(2) e (1)-(3) geram um sistema linear e um modelo em espaços de estados Gaussiano para cada ativo $i$ :

$$
\begin{aligned}
y_{i t} & =C_{t} x_{i t}+\varepsilon_{i t} \\
x_{i t} & =F x_{i t-1}+R u_{i t}
\end{aligned}
$$

onde $C_{t}$ contém os fatores e $x_{i t}$ os pesos dos fatores variantes no tempo, os quais são tratados como estados não observados. A matriz de transição $F$ garante que os estados evoluem de acordo com (2) e (3)-(4) , modelo RW e modelo de aprendizagem, respectivamente. ${ }^{2}$ o termo de erro $u_{t}$ é normalmente distribuído com média zero e matriz de covariância $\Sigma_{u_{i}}, R$ é uma coleção de vetores em que as entradas são 0 ou 1. Este modelo pode ser estimado por ML usando o filtro de Kalman para construir a verossimilhança da função, a qual é maximizada para obter os parâmetros estimados.

$O$ vetor de estados não observados, $x_{i t}$, que no caso do modelo de aprendizagem inclui não apenas $\beta_{i t}$ mas também $B_{i t}$, pode ser estimado condicionado às informações passadas e correntes, $y_{i 1}, \ldots, y_{i t}$, via filtro de Kalman. Como ressaltado por Harvey et alii (1992), o filtro de Kalman é o estimador de mínimo erro quadrático para os estados mesmo quando efeitos $\mathrm{GARCH}$ presentes nos erros são ignorados. Tomando como base $x_{i t \mid t-1}$ e $P_{t \mid t-1}$, quando a observação $y_{i t}$ está disponível, o erro de predição pode ser calculado: $v_{i t}=y_{i t}-f_{t}^{\prime} x_{i t \mid t-1}$. Assim, depois da observação $y_{i t}$, uma inferência mais acurada de $x_{i t \mid t}$ e $P_{t \mid t}$ pode ser obtida:

$$
\begin{gathered}
x_{i t \mid t}=x_{i t \mid t-1}+P_{t \mid t-1} f_{t}^{\prime} \Delta_{t}^{-1} v_{t}, \\
P_{t \mid t}=P_{t \mid t-1}-P_{t \mid t-1} f_{t}^{\prime} \Delta_{t}^{-1} f_{t} P_{t \mid t-1},
\end{gathered}
$$

onde $\Delta_{t}=f_{t}^{\prime} P_{t \mid t-1} f_{t}+\sigma_{v}$ é o erro de predição da matriz de covariância. Uma estimativa do vetor de estados no período $t+1$ condicional a $y_{1}, \ldots, y_{t}$, é dada pelo passo da predição

$$
\begin{gathered}
x_{t+1 \mid t}=F x_{t \mid t}, \\
P_{t+1 \mid t}=F P_{t \mid t} F^{\prime}+R \Sigma_{u_{i}} R^{\prime} .
\end{gathered}
$$

Para uma dada série de tempo $y_{1}, \ldots, y_{T}$, os cálculos do filtro de Kalman são realizados recursivamente para $t=1, \ldots, T$. Por causa da não estacionariedade da equação de transição (2), a inicialização foi implementada usando o filtro de Kalman inicial exato proposto por Koopman (1997). Os parâmetros na matriz de covariância $\Sigma_{u_{i}}$ são tratados como coeficientes desconhecidos, os quais são colocados no vetor de parâmetros $\psi$. A estimação de $\psi$ é baseada em otimização numérica da função de verossimilhança, que é construída via decomposição do erro de predição e dada por: ${ }^{3}$

$$
l(\psi)=-\frac{N T}{2} \log 2 \pi-\frac{1}{2} \sum_{t=1}^{T} \log \left|F_{t}\right|-\frac{1}{2} \sum_{t=1}^{T} v_{t}^{\prime} \log F_{t}^{-1} v_{t} .
$$

Harvey et alii (1992) sugerem que a estimação de $\psi$ por ML ignorando efeitos GARCH nos disturbios é consistente, já que este resultado é uma mera generalização dos resultado apresentado por Weiss (1984) a respeito da estimação de modelos ARMA com erros ARCH via ML.

\footnotetext{
${ }^{2}$ Note que o vetor de estados no modelo de aprendizagem contém não apenas $\beta_{i, t}$ mas também $B_{i t}$, assim, $C_{t}$ deve incluir algumas colunas de zeros.

${ }^{3}$ Para detalhes a respeito da estimação dos parâmetros e estados através do filtro de Kalman, ver Durbin e Koopman (2001). Para uma derivação completa do filtro de Kalman para o modelo CAPM com aprendizagem, ver o Apêndice B em Adrian e Franzoni (2009).
} 


\section{Estimação da Matriz de Covariância Condicional dos Fatores}

Para obter a matriz de covariância condicional dos fatores, $\Omega_{t}$, é empregada uma especificação DCC em (6). A estimação do modelo DCC pode ser convenientemente dividida em uma parte da volatilidade e outra parte da correlação. A parte da volatilidade refere-se à estimação das volatilidades condicionais univariadas dos fatores através de uma especificação do tipo GARCH. Os parâmetros dos modelos de volatilidade univariados são estimados por quase máxima verossimilhança (QML) assumindo inovações Gaussianas. ${ }^{4}$ A parte da correlação refere-se à estimação da matriz de correlação condicional em (7) e (8). Para estimar os parâmetros da parte da correlação, emprega-se o método de verossimilhança composta (CL) proposto por Engle et alii (2008). Como destacaram Engle et alii (2008), o estimador CL oferece estimativas mais acuradas dos parâmetros estimados em comparação com procedimento de dois passos proposto por Engle e Sheppard (2001) e Sheppard (2003), especialmente em problemas com elevada dimensão.

\subsection{Previsão}

As previsões um passo à frente das matrizes de covariância condicionais baseadas no modelo DFGARCH podem ser obtidas como:

$$
H_{t \mid t-1}=\beta_{t \mid t-1} \Omega_{t \mid t-1} \beta_{t \mid t-1}^{\prime}+\Xi_{t \mid t-1},
$$

onde $\beta_{t \mid t-1}, \Omega_{t \mid t-1}$, and $\Xi_{t \mid t-1}$ são, respectivamente, previsões dos pesos dos fatores um passo à frente obtidas de acordo com (2), previsão um passo à frente da matriz de covariância condicional dos fatores calculada conforme (6), e previsões um passo à frente das variâncias condicionais dos resíduos obtidas por um modelo do tipo GARCH e colocadas na matriz diagonal $\Xi_{t \mid t-1}$.

\subsection{Modelos benchmark}

São consideradas quatro especificações alternativas de benchmark para a matriz de covariância condicional dos retornos. O primeiro modelo benchmark é uma versão da especificação presente em (5) com pesos dos fatores constantes ao longo do tempo, ou seja

$$
H_{t}=\beta \Omega_{t} \beta^{\prime}+\Xi_{t},
$$

onde $\beta$ é a estimativa por mínimos quadrados ordinários (OLS) do modelo de regressão $y_{i t}=\beta_{i} f_{t}+\varepsilon_{i t}$.

O segundo benchmark é o modelo GARCH ortogonal (OGARCH) de Alexander e Chibumba (1996) e Alexander (2001),

$$
H_{t}=W \Lambda_{t} W^{\prime},
$$

onde $W$ é uma matriz $N \times K$ dos autovetores dos primeiros $K \leq N$ fatores ortogonais obtidos via análise de componentes principais (PCA) e $\Lambda_{t}$ é uma matriz de covariância diagonal das variâncias condicionais dos componentes principais, i.e. $\Lambda_{t}=\operatorname{diag}\left(h_{P C_{1 t}}, \ldots, h_{P C_{k t}}\right)$ onde $h_{P C_{t}}$ segue um modelo GARCH.

O terceiro benchmark é o modelo de fatores proposto por Chan et alii (1999) (CKL),

$$
H_{t}=\beta_{t} \Gamma_{t} \beta_{t}^{\prime}+\Upsilon_{t},
$$

\footnotetext{
${ }^{4}$ Uma revisão das questões relacionadas à estimação, tais como, escolha dos valores iniciais, algoritmos numéricos, acurácia, bem como propriedades assintóticas são dadas por Berkes et alii (2003), Robinson e Zaffaroni (2006), Francq e Zakoian (2009), e Zivot (2009). É importante observar que mesmo quando a suposição de normalidade é inapropriada, o estimador QML baseado na maximização das verossimilhanças Gaussianas é consistente e assintoticamente Normal, dado que a média condicional e funções de variância do modelo GARCH são corretamente especificadas; ver Bollerslev e Wooldridge (1992).
} 
onde $\Gamma_{t}$ é a matriz de covariância dos fatores do modelo Fama-French-Carhart, calculada usando uma amostra rolling window de 252 dias, i.e. $\Gamma_{t}=\frac{1}{252} \sum_{i=t-252}^{t-1} f_{i} f_{i}^{\prime}$, e $\Upsilon_{t}$ é a matriz diagonal com as variâncias dos resíduos da regressão rolling window dos retornos dos ativos contra os fatores.

Finalmente, o quarto modelo benchmark é o modelo Risk Metrics (RM), o qual consiste de um modelo de média móvel exponencialmente ponderado para modelar as covariâncias condicionais. Nesta abordagem, a matriz de covariância condicional é dada por

$$
H_{t}=(1-\lambda) Y_{t-1} Y_{t-1}^{\prime}+\lambda H_{t-1}
$$

com o valor recomendado para o parâmetro em estimações com dados diários sendo $\lambda=0.94$.

\section{APLICAÇÃO EM OTIMIZAÇÃO DE CARTEIRAS}

O problema da obtenção da CMV pode ser visto como um caso particular do problema da obtenção de carteiras ótimas baseadas em retorno esperado e variância condicional conhecido como média-variância (Markowitz, 1952). Formalmente, a carteira de média-variância pode ser obtida através da solução do problema de maximização da utilidade do investidor com base no trade-off entre risco e retorno 


\subsection{Dados e detalhes da implementação}

O problema de seleção de carteiras é tratado empiricamente usando dados históricos de ações negociadas na BM\&F Bovespa. A base de dados é composta por observações diárias dos preços de 61 ações que fizeram parte do índice Ibovespa de Janeiro de 2000 a dezembro de 2010, perfazendo um total de 2722 observações diárias. Os retornos são calculados como a diferença dos logaritmos dos preços. Os dados foram obtidos do Economática.

As primeiras 1722 observações são usadas para estimar os parâmetros de todos os modelos e obter previsões dentro da amostra, enquanto que as últimas 1000 observações são usadas para obter previsões fora da amostra. As previsões são do tipo não-adaptativas, isto é, os parâmetros estimados no período dentro da amostra foram mantidos fixos no período fora da amostra. A Tabela 1 apresenta estatísticas descritivas da base de dados utilizada para os períodos dentro e fora da amostra. Pode-se observar que o retorno médio é menor e a curtose média mais elevada no período fora da amostra em comparação com o período dentro da amostra. Estas diferenças nos momentos amostrais se devem ao fato de que o período fora da amostra vai de janeiro de 2007 a dezembro de 2010, incluindo a crise financeira deflagrada naquele período.

É importante ressaltar três questões técnicas relativas à implementação dos modelos benchmark. Primeiro, na implementação do modelo CLK foram utilizadas estimações rolling window com 252 observações. Portanto, não foram incluídas as primeiras 252 observações na avaliação do desempenho de todos os modelos. Segundo, a abordagem Risk Metrics não envolve nenhum parâmetro desconhecido, uma vez que é definido $\lambda=0.94$. Finalmente, ao implementar o modelo OGARCH, considera-se diferentes números de fatores. Em particular, o modelo OGARCH é implementado com o número de fatores variando de 1 até $N$. Para facilitar a discussão e exposição dos resultados são apresentados os resultados do modelo OGARCH apenas para a especificação que apresentou melhor desempenho.

\section{Tabela 1: Estatísticas descritivas}

A tabela apresenta estatísticas descritivas para os períodos dentro e fora da amostra e estatísticas descritivas para o conjunto de dados composto por 61 ações que pertenceram ao índice Ibovespa.

\begin{tabular}{lccc}
\hline & $\begin{array}{c}\text { Dentro da amostra } \\
01 / 01 / 2000-09 / 01 / 2007\end{array}$ & & $\begin{array}{c}\text { Fora da amostra } \\
09 / 01 / 2007-31 / 12 / 2010\end{array}$ \\
\cline { 2 - 2 } Número de obs. & 1722 & & 1000 \\
Retorno médio (\%) & 0.068 & 0.042 \\
Desvio padrão & 2.887 & 2.865 \\
Mediana & -0.001 & 0.025 \\
Curtose & 9.166 & 11.110 \\
Assimetria & 0.223 & 0.113 \\
\hline
\end{tabular}

\subsection{Metodologia para avaliação do desempenho}

O desempenho das carteiras otimizadas é avaliada em termos de variância dos retornos da carteira $\left(\widehat{\sigma}^{2}\right)$, índice de Sharpe (IS) e turnover. Estas estatísticas são calculadas como segue: 


$$
\begin{aligned}
\hat{\sigma}^{2} & =\frac{1}{T-1} \sum_{t=1}^{T-1}\left(w_{t}^{\prime} R_{t+1}-\hat{\mu}\right)^{2} \\
I S & =\frac{\hat{\mu}}{\hat{\sigma}}, \text { onde } \hat{\mu}=\frac{1}{T-1} \sum_{t=1}^{T-1} w_{t}^{\prime} R_{t+1} \\
\text { Turnover } & =\frac{1}{T-1} \sum_{t=1}^{T-1} \sum_{j=1}^{N}\left(\left|w_{j, t+1}-w_{j, t}\right|\right),
\end{aligned}
$$

onde $w_{j, t}$ é o peso do ativo $j$ no portfólio no tempo $t+1$ antes do rebalanceamento e $w_{j, t+1}$ é o peso desejado do do ativo $j$ no tempo $t+1$. Como destacado por DeMiguel et alii (2009b), o turnover, como definido acima, pode ser interpretado como a fração média do volume de recursos alocado na carteira que é transacionado em cada período.

Para testar a significância estatística das diferenças entre as variâncias e índices de Sharpe dos retornos de duas carteiras adota-se estratégia similar a DeMiguel et alii (2009a) em que é usado o bootstrap estacionário de Politis e Romano (1994) com $B=1,000$ reamostragens e tamanho de bloco $b=5 .^{5}$ Os $p$-valores do teste foram obtidos usando a metodologia sugerida em Ledoit e Wolf (2008, Remark 3.2).

\subsection{Resultados}

A Tabela 2 apresenta a variância, o índice de Sharpe e o turnover das carteiras considerando períodos dentro da amostra e fora da amostra, para carteiras de variância mínima com restrição de venda a descoberto e irrestritas obtidas com matrizes de covariância geradas pelo modelo DFGARCH proposto por Santos e Moura (2012) com pesos dos fatores variando no tempo, baseado no modelo RW (DFGARCHRW), modelo de aprendizagem (DFGARCH-learning) e para os modelos benchmark. Os modelos benchmark propostos são o modelo com pesos dos fatores invariantes no tempo (DFGARCH-OLS), o modelo OGARCH, o modelo Risck Metrics e o modelo CKL. A tabela traz também os $p$-valores para as diferenças entre as variâncias das carteiras e índice de Sharpe em relação àqueles obtidos com o modelo CKL.

Os resultados mostram que em termos de variância o modelo DFGARCH-RW gera as carteiras ótimas com menor risco, tanto no período dentro da amostra como fora da amostra e também considerando as duas políticas de investimento, seja com restrição de venda a descoberto ou irrestrito. Por exemplo, no período fora da amostra o modelo DFGARCH-RW gera um portfólio com variância de 0.511 no caso irrestrito, a qual é substancialmente (e estatisticamente) menor que a variância das carteiras obtidas com os modelos OGARCH, RiskMetrics e CKL (2.849, 1.560 e 0.874, respectivamente). Podemos observar também que a variância do portfólio obtido com o modelo DFGARCH-RW é menor em comparação às carteiras resultantes dos modelos DFGARCH-learning e DFGARCH-OLS.

Em termos de índice de Sharpe os resultados indicam que o modelo DFGARCH-learning gera melhor desempenho ajustado pelo risco em comparação aos demais modelos, incluindo todos os modelos benchmark. Por exemplo, o modelo DFGARCH-learning apresenta IS de 0.076 para o caso da política de investimentos irrestrito durante o período fora da amostra, enquanto o modelo CKL apresenta IS de 0.027 , sendo as diferenças estatisticamente significantes ao nível de $10 \%$. Em termos de portfólio turnover nota-se que o melhor desempenho foi alcançado pelo modelo CKL em todos os casos, seguido pelos modelos OGARCH e DFGARCH-learning. Pode-se observar também que em todos os casos o modelo

\footnotetext{
${ }^{5}$ Foram realizados extensivos testes de robustez para definir o tamanho do bloco, usando valores para $b$ entre 5 e 250 . Independente do tamanho do bloco, os resultados dos testes para a variância e índice de Sharpe são similares aos reportados aqui.
} 
Tabela 2: Desempenho das carteiras de variância mínima com rebalanceamento diário

A tabela apresenta a média diária da variância da carteira, o índice de Sharpe e portfólio turnover dos porftólios de variância mínima com restrição de venda a descoberto e irrestrito, obtidos com matrizes de covariâncias geradas pelo modelo DFGARCHRW e modelo DFGARCH com aprendizagem. Os modelos benchmark são o DFGARCH-OLS, o modelo OGARCH, modelo RiskMetrics e o modelo CKL. $p$-valores para as diferenças entre as variâncias das carteiras e índices de Sharpe em relação àqueles obtidos com o modelo CKL estão entre parênteses abaixo de cada coeficiente.

\begin{tabular}{|c|c|c|c|c|c|c|}
\hline \multirow{2}{*}{ Dentro da amostra } & Variância & Índice de Sharpe & Turnover & Variância & Índice de Sharpe & Turnover \\
\hline & \multicolumn{3}{|c|}{ Restrito } & \multicolumn{3}{|c|}{ Irrestrito } \\
\hline \multirow[t]{2}{*}{ DFGARCH-learning } & 0.721 & 0.141 & 0.086 & 0.642 & 0.179 & 0.123 \\
\hline & $(0.314)$ & $(0.084)$ & & $(0.870)$ & $(0.474)$ & \\
\hline \multirow[t]{2}{*}{ DFGARCH-RW } & 0.431 & 0.119 & 0.105 & 0.391 & 0.162 & 0.167 \\
\hline & $(0.000)$ & $(0.844)$ & & $(0.000)$ & $(0.763)$ & \\
\hline \multirow[t]{2}{*}{ DFGARCH-OLS } & 0.642 & 0.097 & 0.093 & 0.586 & 0.104 & 0.159 \\
\hline & $(0.043)$ & $(0.195)$ & & $(0.026)$ & $(0.000)$ & \\
\hline \multirow[t]{2}{*}{ OGARCH } & 1.910 & 0.068 & 0.032 & 2.116 & 0.067 & 0.070 \\
\hline & $(0.000)$ & $(0.026)$ & & $(0.000)$ & $(0.000)$ & \\
\hline \multirow[t]{2}{*}{ RiskMetrics } & 0.958 & 0.074 & 0.173 & 1.858 & 0.074 & 0.929 \\
\hline & $(0.212)$ & $(0.027)$ & & $(0.000)$ & $(0.000)$ & \\
\hline \multirow[t]{2}{*}{ CKL } & 0.693 & 0.117 & 0.024 & 0.636 & 0.166 & 0.043 \\
\hline & $(1.000)$ & $(1.000)$ & & $(1.000)$ & $(1.000)$ & \\
\hline Fora da amostra & & Restrito & & & Irrestrito & \\
\hline \multirow[t]{2}{*}{ DFGARCH-learning } & 1.645 & 0.067 & 0.094 & 1.461 & 0.076 & 0.139 \\
\hline & $(0.010)$ & $(0.058)$ & & $(0.000)$ & $(0.098)$ & \\
\hline \multirow[t]{2}{*}{ DFGARCH-RW } & 0.558 & 0.052 & 0.128 & 0.511 & 0.042 & 0.186 \\
\hline & $(0.000)$ & $(0.148)$ & & $(0.000)$ & $(0.550)$ & \\
\hline \multirow[t]{2}{*}{ DFGARCH-OLS } & 1.051 & 0.056 & 0.108 & 0.836 & 0.070 & 0.179 \\
\hline & $(0.883)$ & $(0.100)$ & & $(0.655)$ & $(0.120)$ & \\
\hline \multirow[t]{2}{*}{ OGARCH } & 2.553 & 0.056 & 0.108 & 2.849 & 0.024 & 0.063 \\
\hline & $(0.000)$ & $(0.700)$ & & $(0.000)$ & $(0.881)$ & \\
\hline \multirow[t]{2}{*}{ RiskMetrics } & 0.806 & 0.053 & 0.171 & 1.560 & 0.031 & 0.938 \\
\hline & $(0.020)$ & $(0.186)$ & & $(0.000)$ & $(0.868)$ & \\
\hline \multirow[t]{2}{*}{ CKL } & 1.083 & 0.015 & 0.026 & 0.874 & 0.027 & 0.048 \\
\hline & $(1.000)$ & $(0.000)$ & & $(1.000)$ & $(1.000)$ & \\
\hline
\end{tabular}

DFGARCH-learning alcança menor turnover em comparação com os modelos DFGARCH-OLS e DFGARCHRW. Estes resultados sugerem que os pesos dos fatores com aprendizagem resultam em carteiras ótimas com menor variância e também com menor turnover. Finalmente, obseva-se que o portfólio turnover associado à política de investimento com restrição de venda à descoberto tende a ser menor quanto comparado com o caso irrestrito. Este resultado está em linha com resultados empíricos anteriores na literatura, tais como DeMiguel et alii (2009a) e Santos e Moura (2012).

Para ilustrar ainda mais os resultados, na Figura 1 é apresentado o diagrama de caixas (boxplot) dos retornos das CMV para os períodos dentro da amostra e fora da amostra, considerando políticas de investime nto com restrição à venda a descoberto e irrestrita. A figura mostra que a dispersão dos retornos do portfólio obtido com o modelo DFGARCH-RW é substancialmente menor em comparação com os demais modelos em todos os casos, o que corrobora com os resultados da Tabela 2. Podese observar também que a dispersão dos retornos do portfólio durante o período fora da amostra é

RBE Rio de Janeiro v. 67 n. 1/p. 45-65 Jan-Mar 2013 
maior quando comparado com o período dentro da amostra, o que reflete o impacto da crise financeira mundial deflagrada em 2007-2008.

Figura 1: Boxplot dos retornos das carteiras
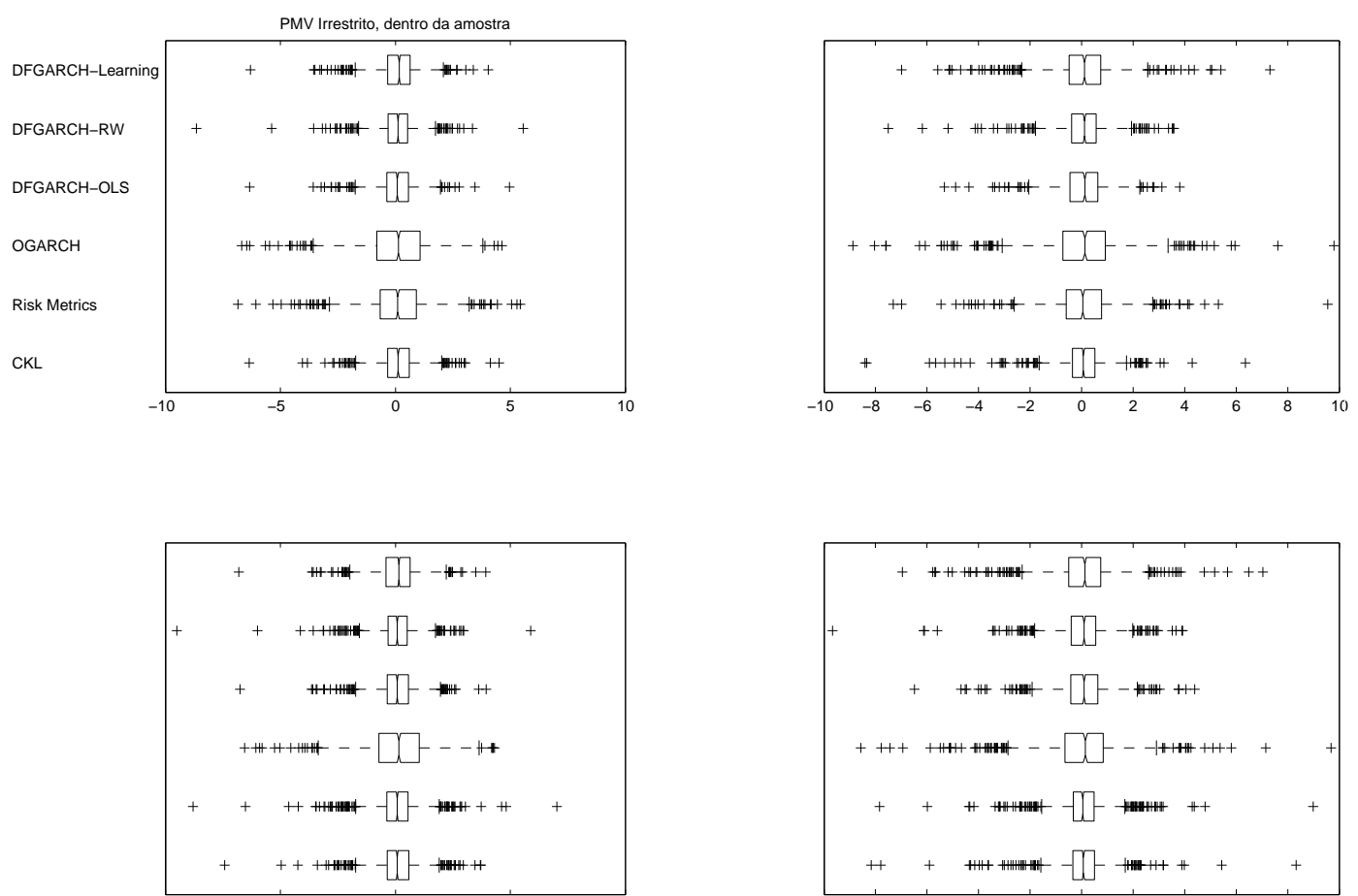
Tabela 3: Desempenho das carteiras de variância mínima com rebalanceamento semanal

A tabela apresenta a média diária da variância da carteira, o índice de Sharpe e portfólio turnover dos porftólios de variância mínima com restrição de venda a descoberto e irrestrito, obtidos com matrizes de covariâncias geradas pelo modelo DFGARCHRW e modelo DFGARCH com aprendizagem. Os modelos benchmark são o DFGARCH-OLS, o modelo OGARCH, modelo RiskMetrics e o modelo CKL. $p$-valores para as diferenças entre as variâncias das carteiras e índices de Sharpe em relação àqueles obtidos com o modelo CKL estão entre parênteses abaixo de cada coeficiente.

\begin{tabular}{|c|c|c|c|c|c|c|}
\hline \multirow{2}{*}{ Dentro da amostra } & Variância & Índice de Sharpe & Turnover & Variância & Índice de Sharpe & Turnover \\
\hline & \multicolumn{3}{|c|}{ Restrito } & \multicolumn{3}{|c|}{ Irrestrito } \\
\hline \multirow[t]{2}{*}{ DFGARCH-learning } & 0.757 & 0.132 & 0.040 & 0.681 & 0.166 & 0.055 \\
\hline & $(0.036)$ & $(0.308)$ & & $(0.158)$ & $(0.907)$ & \\
\hline \multirow[t]{2}{*}{ DFGARCH-RW } & 0.734 & 0.095 & 0.050 & 0.620 & 0.141 & 0.076 \\
\hline & $(0.824)$ & $(0.365)$ & & $(0.701)$ & $(0.273)$ & \\
\hline \multirow[t]{2}{*}{ DFGARCH-OLS } & 0.664 & 0.106 & 0.043 & 0.604 & 0.113 & 0.072 \\
\hline & $(0.233)$ & $(0.393)$ & & $(0.175)$ & $(0.002)$ & \\
\hline \multirow[t]{2}{*}{ OGARCH } & 1.910 & 0.068 & 0.014 & 2.117 & 0.067 & 0.033 \\
\hline & $(0.000)$ & $(0.018)$ & & $(0.000)$ & $(0.000)$ & \\
\hline \multirow[t]{2}{*}{ RiskMetrics } & 1.075 & 0.069 & 0.091 & 1.973 & 0.066 & 0.475 \\
\hline & $(0.106)$ & $(0.024)$ & & $(0.000)$ & $(0.000)$ & \\
\hline \multirow[t]{2}{*}{ CKL } & 0.698 & 0.118 & 0.013 & 0.640 & 0.167 & 0.023 \\
\hline & $(1.000)$ & $(1.000)$ & & $(1.000)$ & $(1.000)$ & \\
\hline Fora da amostra & & Restrito & & & Irrestrito & \\
\hline \multirow[t]{2}{*}{ DFGARCH-learning } & 1.681 & 0.053 & 0.042 & 1.531 & 0.057 & 0.061 \\
\hline & $(0.022)$ & $(0.144)$ & & $(0.000)$ & $(0.310)$ & \\
\hline \multirow[t]{2}{*}{ DFGARCH-RW } & 0.821 & 0.035 & 0.059 & 0.710 & 0.038 & 0.085 \\
\hline & $(0.049)$ & $(0.352)$ & & $(0.069)$ & $(0.498)$ & \\
\hline \multirow[t]{2}{*}{ DFGARCH-OLS } & 1.056 & 0.058 & 0.050 & 0.865 & 0.072 & 0.081 \\
\hline & $(0.745)$ & $(0.108)$ & & $(0.723)$ & $(0.080)$ & \\
\hline \multirow[t]{2}{*}{ OGARCH } & 2.553 & 0.026 & 0.013 & 2.854 & 0.022 & 0.031 \\
\hline & $(0.000)$ & $(0.642)$ & & $(0.000)$ & $(0.945)$ & \\
\hline \multirow[t]{2}{*}{ RiskMetrics } & 0.888 & 0.041 & 0.093 & 1.583 & 0.019 & 0.486 \\
\hline & $(0.075)$ & $(0.310)$ & & $(0.000)$ & $(0.873)$ & \\
\hline \multirow[t]{2}{*}{ CKL } & 1.122 & 0.012 & 0.016 & 0.905 & 0.024 & 0.027 \\
\hline & $(1.000)$ & $(1.000)$ & & $(1.000)$ & $(1.000)$ & \\
\hline
\end{tabular}

\section{CONSIDERAÇÕES FINAIS}

Os modelos de fatores estão atualmente estabelecidos como uma alternativa promissora para reduzir problemas de dimensionalidade e o custo computacional do processo de estimação quando o objetivo é obter matrizes de covariâncias de carteiras contendo um grande número de ativos. Neste artigo, é empregada uma abordagem recentemente proposta por Santos e Moura (2012) para obter matrizes de covariâncias condicionais baseado em um modelo de fatores que estende especificações econométricas anteriores. A abordagem permite uma especificação mais parcimoniosa para os fatores comuns e especificações alternativas para os ativos individuais do portfolio. Além disso, os pesos dos fatores são tratados como variáveis latentes variando ao longo do tempo e são consideradas dinâmicas baseadas em recentes desenvolvimentos na teoria de apreçamento de ativos.

O modelo é utilizado para obter previsões um passo à frente para dentro e fora da amostra da matriz de covariância condicional de 61 ativos que pertenceram ao índice Ibovespa durante o período 
Tabela 4: Desempenho das carteiras de variância mínima com rebalanceamento mensal

A tabela apresenta a média diária da variância da carteira, o índice de Sharpe e portfólio turnover dos porftólios de variância mínima com restrição de venda a descoberto e irrestrito, obtidos com matrizes de covariâncias geradas pelo modelo DFGARCHRW e modelo DFGARCH com aprendizagem. Os modelos benchmark são o DFGARCH-OLS, o modelo OGARCH, modelo RiskMetrics e o modelo CKL. $p$-valores para as diferenças entre as variâncias das carteiras e índices de Sharpe em relação àqueles obtidos com o modelo CKL estão entre parênteses abaixo de cada coeficiente.

\begin{tabular}{|c|c|c|c|c|c|c|}
\hline \multirow{2}{*}{ Dentro da amostra } & Variância & Índice de Sharpe & Turnover & Variância & Índice de Sharpe & Turnover \\
\hline & \multicolumn{3}{|c|}{ Restrito } & \multicolumn{3}{|c|}{ Irrestrito } \\
\hline \multirow[t]{2}{*}{ DFGARCH-learning } & 0.780 & 0.124 & 0.016 & 0.710 & 0.159 & 0.021 \\
\hline & $(0.116)$ & $(0.330)$ & & $(0.230)$ & $(0.996)$ & \\
\hline \multirow[t]{2}{*}{ DFGARCH-RW } & 0.695 & 0.095 & 0.021 & 0.632 & 0.136 & 0.031 \\
\hline & $(0.575)$ & $(0.523)$ & & $(0.519)$ & $(0.323)$ & \\
\hline \multirow[t]{2}{*}{ DFGARCH-OLS } & 0.688 & 0.108 & 0.017 & 0.633 & 0.115 & 0.028 \\
\hline & (0.149) & $(0.871)$ & & $(0.106)$ & $(0.012)$ & \\
\hline \multirow[t]{2}{*}{ OGARCH } & 1.912 & 0.068 & 0.006 & 2.125 & 0.065 & 0.014 \\
\hline & $(0.000)$ & $(0.065)$ & & $(0.000)$ & $(0.000)$ & \\
\hline \multirow[t]{2}{*}{ RiskMetrics } & 0.902 & 0.077 & 0.041 & 1.796 & 0.092 & 0.209 \\
\hline & $(0.006)$ & $(0.126)$ & & $(0.000)$ & $(0.006)$ & \\
\hline \multirow[t]{2}{*}{ CKL } & 0.733 & 0.109 & 0.008 & 0.674 & 0.159 & 0.014 \\
\hline & $(1.000)$ & $(1.000)$ & & $(1.000)$ & $(1.000)$ & \\
\hline Fora da amostra & & Restrito & & & Irrestrito & \\
\hline \multirow[t]{2}{*}{ DFGARCH-learning } & 1.700 & 0.049 & 0.017 & 1.562 & 0.051 & 0.0239 \\
\hline & $(0.076)$ & $(0.278)$ & & $(0.004)$ & $(0.576)$ & \\
\hline \multirow[t]{2}{*}{ DFGARCH-RW } & 1.048 & 0.044 & 0.026 & 0.911 & 0.055 & 0.037 \\
\hline & $(0.255)$ & $(0.226)$ & & $(0.635)$ & $(0.348)$ & \\
\hline \multirow[t]{2}{*}{ DFGARCH-OLS } & 1.050 & 0.064 & 0.021 & 0.892 & 0.077 & 0.032 \\
\hline & $(0.345)$ & $(0.072)$ & & $(0.521)$ & $(0.091)$ & \\
\hline \multirow[t]{2}{*}{ OGARCH } & 2.552 & 0.026 & 0.006 & 2.854 & 0.023 & 0.014 \\
\hline & $(0.000)$ & $(0.798)$ & & $(0.000)$ & $(0.737)$ & \\
\hline \multirow[t]{2}{*}{ RiskMetrics } & 1.310 & 0.037 & 0.047 & 1.997 & 0.032 & 0.224 \\
\hline & $(0.628)$ & $(0.452)$ & & $(0.000)$ & $(0.919)$ & \\
\hline \multirow[t]{2}{*}{ CKL } & 1.243 & 0.020 & 0.010 & 0.979 & 0.034 & 0.017 \\
\hline & $(1.000)$ & $(1.000)$ & & $(1.000)$ & $(1.000)$ & \\
\hline
\end{tabular}

amostral, e posteriormente usa-se as matrizes estimadas para obter portfólios de mínima variância com restrição de venda à descoberto e irrestrito. O desempenho do modelo proposto é comparado àquele de modelos benchmark alternativos, incluindo modelos fatoriais existentes. Os resultados indicam que o modelo proposto entrega carteiras com menor risco em comparação aos modelos benchmark, bem como melhor desempenho ajustado pelo risco. Além disso, os resultados são robustos com relação a frequência de rebalanceamento das carteiras ótimas. 


\section{BIBLIOGRAFIA}

Adrian, T. \& Franzoni, F. (2009). Learning about beta: Time-varying factor loadings, expected returns, and the conditional CAPM. Journal of Empirical Finance, 16(4):537-556.

Aguilar, O. \& West, M. (2000). Bayesian dynamic factor models and portfolio allocation. Journal of Business and Economic Statistics, 18(3):338-357.

Alexander, C. (2001). Orthogonal GARCH. Mastering Risk, 2:21-38.

Alexander, C. \& Chibumba, A. (1996). Multivariate orthogonal factor GARCH. Working paper, Mathematics Department, University of Sussex.

Ang, A. \& Chen, J. (2007). CAPM over the long run: 1926-2001. Journal of Empirical Finance, 14(1):1-40.

Bauwens, L., Laurent, S., \& Rombouts, J. (2006). Multivariate GARCH models: A survey. Journal of Applied Econometrics, 21(1):79-109.

Berkes, I., Horváth, L., \& Kokoszka, P. (2003). GARCH processes: Structure and estimation. Bernoulli, 9(2):201-227.

Best, M. \& Grauer, R. (1991). On the sensitivity of mean-variance-efficient portfolios to changes in asset means: Some analytical and computational results. Review of Financial Studies, 4(2):315-342.

Bollerslev, T. (1986). Generalized autoregressive conditional heteroskedasticity. Journal of Econometrics, 31(3):307-327.

Bollerslev, T., Engle, R., \& Wooldridge, J. (1988). A capital asset pricing model with time-varying covariances. The Journal of Political Economy, 96(1):116.

Bollerslev, T. \& Wooldridge, J. (1992). Quasi-maximum likelihood estimation and inference in dynamic models with time-varying covariances. Econometric Reviews, 11(2):143-172.

Brav, A., Geczy, C., \& Gompers, P. A. (2000). Is the abnormal return following equity issuance anomalous? Journal of Financial Economics, 56(2):209-249.

Cappiello, L., Engle, R., \& Sheppard, K. (2006). Asymmetric dynamics in the correlations of global equity and bond returns. Journal of Financial Econometrics, 4(4):537-572.

Carhart, M. M. (1997). On persistence in mutual fund performance. The Journal of Finance, 52(1):57-82.

Ceria, S. \& Stubbs, R. (2006). Incorporating estimation errors into portfolio selection: Robust portfolio construction. Journal of Asset Management, 7(2):109-127.

Chan, L., Karceski, J., \& Lakonishok, J. (1999). On portfolio optimization: Forecasting covariances and choosing the risk model. Review of Financial Studies, 12(5):937-74.

Chib, S., Omori, Y., \& Asai, M. (2009). Multivariate stochastic volatility. Springer Verlag.

Chung, Y. \& Schill, J. (2006). Asset pricing when returns are nonnormal: Fama-French factors versus higher-order systematic co-moments. Journal of Business, 79(2):923-940.

Costa Jr, N. \& Neves, M. (2000). Variáveis fundamentalistas e os retornos das ações. Revista Brasileira de Economia, 54(1):123-137.

DeMiguel, V., Garlappi, L., Nogales, F., \& Uppal, R. (2009a). A generalized approach to portfolio optimization: Improving performance by constraining portfolio norms. Management Science, 55(5):798-812.

RBE Rio de Janeiro v. 67 n. 1 / p. 45-65 Jan-Mar 2013 
DeMiguel, V., Garlappi, L., \& Uppal, R. (2009b). Optimal versus naive diversification: How inefficient is the 1/N portfolio strategy? Review of Financial Studies, 22(5):1915-1953.

Ding, Z., Granger, C., \& Engle, R. (1993). A long memory property of stock returns and a new model. Journal of Empirical Finance, 1(1):83-106.

Durbin, J. \& Koopman, S. J. (2001). Time Series Analysis by State Space Methods. Oxford University Press, 2 edition.

Ekbo, B. E., Masulis, R. W., \& Norli, O. (2000). Seasoned public offerings: Resolution of the 'new issues puzzle'. Journal of Financial Economics, 56(2):251-291.

Engle, R. (1990). Stock volatility and the crash of '87: Discussion. The Review of Financial Studies, 3(1):103-106.

Engle, R. (2002). Dynamic conditional correlation: A simple class of multivariate generalized autoregressive conditional heteroskedasticity models. Journal of Business \& Economic Statistics, 20(3):339-350.

Engle, R. \& Kroner, K. (1995). Multivariate simultaneous generalized ARCH. Econometric Theory, 11(1):122-50.

Engle, R. \& Ng, V. (1993). Measuring and Testing the Impact of News on Volatility. Journal of Finance, 48(5):1749-78.

Engle, R., Ng, V., \& Rothschild, M. (1990). Asset pricing with a factor-arch covariance structure: Empirical estimates for treasury bills. Journal of Econometrics, 45(1-2):213-237.

Engle, R., Shephard, N., \& Sheppard, K. (2008). Fitting vast dimensional time-varying covariance models. Discussion Paper Series n.403, Department of Economics, University of Oxford.

Engle, R. \& Sheppard, K. (2001). Theoretical and empirical properties of dynamic conditional correlation multivariate GARCH. NBER Working Paper W8554.

Engle, R. \& Sheppard, K. (2008). Evaluating the specification of covariance models for large portfolios. Working Paper, Department of Economics, University of Oxford.

Fama, E. \& French, K. (1993). Common risk factors in the returns on stocks and bonds. Journal of Financial Economics, 33(1):3-56.

Fama, E. F. \& French, K. (1992). The cross-section of expected stock returns. Journal of Finance, 47(2):427465.

Fama, E. F. \& French, K. (1996). Multifactor explanation of asset pricing anomalies. Journal of Finance, 56(1):55-84.

Francq, C. \& Zakoian, J. (2009). A tour in the asymptotic theory of GARCH estimation. In Andersen, T., Davis, R., Kreiss, J.-P., \& Mikosch, T., editors, Handbook of Financial Time Series. Springer Verlag.

Glosten, L., Jagannathan, R., \& Runkle, D. (1993). On the relation between the expected value and the volatility of the nominal excess return on stocks. Journal of Finance, 48:1779-1801.

Han, Y. (2006). Asset allocation with a high dimensional latent factor stochastic volatility model. Review of Financial Studies, 19(1):237-271.

Harvey, A., Ruiz, E., \& Sentana, E. (1992). Unobserved component time series models with ARCH disturbances. Journal of Econometrics, 52(1):129-157. 
Harvey, A., Ruiz, E., \& Shephard, N. (1994). Multivariate stochastic variance models. Review of Economic Studies, 61(2):247-64.

Jagannathan, R. \& Ma, T. (2003). Risk reduction in large portfolios: Why imposing the wrong constraints helps. The Journal of Finance, 58(4):1651-1684.

Jegadeesh, N. (2000). Long-term performance of seasoned equity offerings: Benchmark errors and biases in expectations. Financial Management, pages 5-30.

Jegadeesh, N. \& Titman, S. (1993). Returns to buying winners and selling losers: Implications for stock market efficiency. Journal of Finance, 48:65-91.

Jostova, G. \& Philipov, A. (2005). Bayesian analysis of stochastic betas. Journal of Financial and Quantitative Analysis, 40(4):747.

Koopman, S. J. (1997). Exact initial Kalman filtering and smoothing for nonstationary time series models. Journal of the American Statistical Association, 92(440):1630-1638.

Ledoit, O. \& Wolf, M. (2004). Honey, I Shrunk the Sample Covariance Matrix. Journal of Portfolio Management, 30(4):110-119.

Ledoit, O. \& Wolf, M. (2008). Robust performance hypothesis testing with the Sharpe ratio. Journal of Empirical Finance, 15(5):850-859.

Liew, J. \& Vassalou, M. (2000). Can book-to-market, size and momentum be risk factors that predict economic growth? Journal of Financial Economics, 57(2):221-245.

Malaga, F. \& Securato, J. R. (2004). Aplicação do modelo de três fatores de Fama e French no mercado acionário brasileiro - Um estudo empírico do período 1995 a 2003. In Anais do Encontro Anual da ENANPAD.

Markowitz, H. (1952). Portfolio Selection. Journal of Finance, 7(1):77-91.

Mendes, B. \& Leal, R. (2005). Robust multivariate modeling in finance. International Journal of Managerial Finance, 1(2):95-106.

Merton, R. (1980). On estimating the expected return on the market: An exploratory investigation. Journal of Financial Economics, 8(4):323-361.

Michaud, R. (1989). The Markowitz Optimization Enigma: Is Optimized Optimal. Financial Analysts Journal, 45(1):31-42.

Mussa, A., Santos, J. O., \& Famá, R. (2012). A adição do fator de risco momento ao modelo de precificação de ativos dos tês fatores de Fama \& French aplicado ao mercado acionário brasileiro. Revista de Gestão, 19(3):431-447.

Nelson, D. (1991). Conditional heteroskedasticity in asset returns: A new approach. Econometrica, 59(2):347-370.

Politis, D. \& Romano, J. (1994). The stationary bootstrap. Journal of the American Statistical Association, 89(428):1303-1313.

Robinson, P. \& Zaffaroni, P. (2006). Pseudo-maximum likelihood estimation of $\mathrm{ARCH}(\infty)$ models. The Annals of Statistics, 34(3):1049-1074. 
Rostagno, L. M., Soares, R. O., \& Soares, K. T. C. (2006). Estratégias de valor e de crescimento em ações na Bovespa: Uma análise de sete indicadores relacionados ao risco. Revista Contabilidade \& Finanças, 17(42):7-21.

Santos, A. A. P. \& Moura, G. V. (2012). Dynamic factor multivariate GARCH model. Computational Statistics E Data Analysis, no prelo.

Santos, A. A. P. \& Tessari, C. (2012). Técnicas quantitativas de otimização de carteiras aplicadas ao mercado de ações brasileiro. Revista Brasileira de Finanças, 10(3):369-393.

Securato, J. R. \& Rogers, P. (2009). Estudo comparativo no mercado brasileiro do capital asset princing model (CAPM), modelo 3-fatores de Fama e French e reward beta approach. Revista de Administração Contemporânea, 3(1):159-159.

Sheppard, K. (2003). Multi-step estimation of multivariate GARCH models. In Proceedings of the International ICSC. Symposium: Advanced Computing in Financial Markets.

Silvennoinen, A. \& Teräsvirta, T. (2009). Multivariate GARCH models. In Andersen, T., Davis, R., Kreiss, J.-P., \& Mikosch, T., editors, Handbook of Financial Time Series. Springer Verlag.

Thomé Neto, C., Leal, R., \& Almeida, V. (2011). Um índice de mínima variância de ações brasileiras. Economia Aplicada, 15(4):535-557.

Vrontos, I., Dellaportas, P., \& Politis, D. (2003). A full-factor multivariate GARCH model. The Econometrics Journal, 6(2):312-334.

Weiss, A. (1984). ARMA models with ARCH errors. Journal of Time Series Analysis, 5(2):129-143.

Zakoian, J. (1994). Threshold heteroskedastic models. Journal of Economic Dynamics and Control, 18(5):931-955.

Zivot, E. (2009). Practical issues in the analysis of univariate GARCH models. In Andersen, T., Davis, R., Kreiss, J.-P., \& Mikosch, T., editors, Handbook of Financial Time Series. Springer Verlag. 


\section{APÊNDICE 1: CONSTRUÇÃO DOS FATORES DE FAMA-FRENCH-CARHART}

\section{Base de Dados}

A base de dados empregada na construção dos fatores consiste de observações diárias dos preços de fechamento de 61 ações que fizeram parte do Índice Ibovespa durante o período de Janeiro de 2000 a Dezembro de 2010, perfazendo um total de 2.722 observações diárias. Todos os dados referentes a valor contábil, valor de mercado e liquidez são obtidos através do sistema de informação Economática. As séries de preços de fechamento diário foram obtidas do sistema Bloomberg e são corrigidas para proventos e dividendos.

Inicialmente foram consideradas todas as ações que fizeram parte do índice Ibovespa durante o período analisado, porém, após aplicados os critérios de exclusão listados abaixo restaram 61 ações na base de dados. Foram efetuadas as seguintes exclusões: ${ }^{6}$

1. Foram excluídas ações que não apresentavam cotações diárias consecutivas para o período de 12 meses anteriores ou 12 meses posteriores ao de formação das carteiras. Os 12 meses anteriores são necessários para o cálculo do fator momento e os posteriores para o cálculo do retorno das ações, que foram utilizados para a obtenção dos prêmios pelos fatores de risco, bem como dos retornos das carteiras;

2. Ações sem valor de mercado em 31 de dezembro e 30 de junho, com tolerância de 21 dias;

3. Ações de empresas que não possuíam Patrimônio Líquido positivo em dezembro;

4. Ações de empresas financeiras, devido a seu alto grau de endividamento, característico do setor. A exclusão decorre da influência que o endividamento tem sobre o índice BE/ME e do fato do endividamento de empresas financeiras não ter o mesmo significado do endividamento de empresas não-financeiras.

5. Além disso, para que não houvessem distorções do índice $B E / M E$ em empresas que possuíam ações de classe ON e PN, o valor de mercado para cálculo do índice foi obtido pelo somatório dos valores de mercado das ações $\mathrm{ON}$ e PN, mesmo que uma das duas classes de ações não tenha permanecido na amostra. Caso a ação não tenha apresentado valor de mercado em junho, com tolerância de 21 dias, para uma das duas classes de ações, ambas foram excluídas do estudo.

\section{Construção dos Fatores}

A metodologia empregada para construção dos fatores do mercado, tamanho (valor de mercado determinado pela multiplicação entre o preço de fechamento da ação no período $t$ e o número de ações existentes no período $t$ ), índice $\mathrm{BE} / \mathrm{ME}$ (índice de valor patrimonial da ação/preço - determinado pela divisão entre o valor patrimonial da ação em dezembro do ano anterior e o preço desta ação no período t) e momento foi a metodologia padrão utilizada por Fama \& French (1993) na construção do modelo dos três fatores, similar ao procedimento empregado em Costa Jr e Neves (2000) e Mussa et alii (2012). ${ }^{7}$ Os retornos diários de cada ação foram calculados pela expressão $R_{t}=\ln \left(P_{t} / P_{t-1}\right)$, e os retornos das carteiras foram determinados pela média aritimética das ações componentes.

\footnotetext{
${ }^{6} \mathrm{O}$ critério para exclusões seguiu Mussa et alii (2012).

${ }^{7}$ Vários autores aplicaram o modelo de fatores de Fama \& French a dados do mercado de ações brasileiro, por exemplo, Costa Jr e Neves (2000) analisaram empresas negociadas em bolsa no período entre 1987 e 1996 e encontraram evidências de retornos mais altos para ações de valor e para empresas menores, resultados similares aos encontrados em Rostagno et alii (2006), Securato e Rogers (2009) e Mussa et alii (2012).
} 
As seis carteiras usadas para estimar os fatores $S M B$ e $H M L$ foram construídas em cada final de junho, conforme Fama e French (1992), os valores de mercados neste mês já refletem todas as informações contábeis anteriores. Em junho do ano corrente as ações foram ordenadas pelo valor de mercado e divididas pela mediana em dois grupos: B (Big) e S (Small). Para cada um desses grupos ordenou-se as ações pelo índice BE/ME, a amostra foi então separada em três grupos: $30 \%$ inferiores (Low), $40 \%$ médios (Medium) e 30\% superiores (High), de acordo com o valor do índice BE/ME. Dessa forma, foram construídas 6 carteiras baseadas no tamanho e índice book-to-market com aproximadamente o mesmo número de ações.

O fator $S M B$ é a média do retorno das três carteiras de ações de empresas pequenas menos a média do retorno das três carteiras de ações de empresas grandes:

$S M B=\frac{1}{3}($ Small Value + Small Neutral + Small Growth $)-\frac{1}{3}($ Big Value + Big Neutral + Big Growth $)$.

O fator $H M L$ é a média do retorno das duas carteiras de ações de empresas com alto BE/ME menos o retorno das duas carteiras de ações de empresas com baixo BE/ME:

$$
H M L=\frac{1}{2}(\text { Small Value }+ \text { Big Value })-\frac{1}{3}(\text { Small Growth }+ \text { Big Growth }) .
$$

Também em junho de cada ano, todas as ações foram ordenadas de acordo com o retorno acumulado no período entre os meses $t-2$ e $t-12$. Desta forma, foi considerada a estratégia de momento de um ano proposta por Jegadeesh e Titman (1993) e Carhart (1997), calculada de acordo com o desempenho dos últimos doze meses, desconsiderando o mês mais recente. Na seqüência, a amostra foi separada pelo valor mediano em dois grupos: Losers (Los) e Winners (Win), contendo as empresas de piores e melhores retornos históricos acumulados, respectivamente. A expressão que representa o cálculo do fator de risco momento é:

$$
P R 1 Y R=R_{W i n}-R_{L o s}
$$

onde $R_{W i n}$ é o retorno médio diário das ações Winners e $R_{L o s}$ é o retorno médio diário das ações Losers.

O fator de risco do mercado $R_{m}-R_{f}$ é o excesso de retorno ponderado pelo valor de mercado (capitalização a mercado) de todas as ações que fizeram parte do índice Ibovespa durante o período amostral sobre a taxa de juros do CDI diário. 


\section{APÊNDICE 2: MODELOS DE VOLATILIDADE UNIVARIADOS}

Neste Apêndice descrevemos as especificações GARCH univariadas que foram usadas para modelar a variância condicional dos fatores e variância condicional dos resíduos do modelo de fatores.

GARCH:

$$
\sigma_{t}^{2}=\omega+\alpha \epsilon_{t-1}^{2}+\beta \sigma_{t-1}^{2}
$$

Glosten-Jagannathan-Runkle GARCH (GJR-GARCH):

$$
\sigma_{t}^{2}=\omega+\alpha \epsilon_{t-1}^{2}+\gamma I\left[\epsilon_{t-1}<0\right] \epsilon_{t-1}^{2}+\beta \sigma_{t-1}^{2}
$$

Exponential GARCH (EGARCH):

$$
\ln \left(\sigma_{t}^{2}\right)=\omega+\alpha \frac{\left|\epsilon_{t-1}\right|}{\sqrt{\sigma_{t-1}^{2}}}+\gamma \frac{\epsilon_{t-1}}{\sqrt{\sigma_{t-1}^{2}}}+\beta \sigma_{t-1}^{2}
$$

Threshold GARCH (TGARCH):

$$
\sigma_{t}=\omega+\alpha\left|\epsilon_{t-1}\right|+\gamma I\left[\epsilon_{t-1}<0\right]\left|\epsilon_{t-1}\right|+\beta \sigma_{t-1}
$$

Asymmetric power GARCH (APARCH):

$$
\sigma_{t}^{\lambda}=\omega+\alpha\left(\left|\epsilon_{t-1}\right|+\gamma \epsilon_{t-1}\right)^{\lambda}+\beta \sigma_{t-1}^{\lambda}
$$

Asymmetric GARCH (AGARCH):

$$
\sigma_{t}^{2}=\omega+\alpha\left(\epsilon_{t-1}+\gamma\right)^{2}+\beta \sigma_{t-1}^{2}
$$

Nonlinear asymmetric GARCH (NAGARCH):

$$
\sigma_{t}^{2}=\omega+\alpha\left(\epsilon_{t-1}+\gamma \sqrt{\sigma_{t-1}^{2}}\right)^{2}+\beta \sigma_{t-1}^{2}
$$

